Preprint typeset in JHEP style - HYPER VERSION

FERMILAB-PUB-03/245-T

SU-ITP-03/25-T

\title{
The Higgs Mass Bound in Gauge Extensions of the Minimal Supersymmetric Standard Model
}

\author{
Puneet Batra* \\ Department of Physics, Stanford University, Stanford, CA 94305 \\ Antonio Delgado, David E. Kaplan \\ Department of Physics and Astronomy, Johns Hopkins University, Baltimore, MD 21218
}

\author{
Tim M.P. Tait \\ Fermi National Accelerator Laboratory, Batavia, IL 60510
}

\begin{abstract}
The minimal supersymmetric standard model, and extensions, have stringent upper bounds on the mass of the lightest Higgs boson if perturbativity up to the Planck scale is assumed. We argue that these bounds are softened tremendously if the Higgs is charged under an asymptotically free gauge group. We present a model with an additional $S U(2)$ gauge group which easily produces Higgs masses above $200 \mathrm{GeV}$ while avoiding electroweak constraints. If one allows some fine-tuning of the high-scale value of the gauge coupling, Higgs masses greater than $350 \mathrm{GeV}$ are achieved. Unification of couplings is predicted to similar accuracy as in the minimal supersymmetric standard model with only small deviations at the two-loop level.
\end{abstract}

KEYWORDs: aft, exs, suy.

${ }^{*}$ Present Address: Department of Physics and Astronomy, Johns Hopkins University, Baltimore, MD 21218 


\section{Introduction}

The minimal supersymmetric standard model (MSSM) is perhaps the best motivated example of new physics at the weak-scale. Among its virtues are its elegant explanation of the stabilization of the electroweak scale, its dramatic prediction of gauge coupling unification and its low impact on electroweak precision measurements. In fact, the MSSM prediction of a light Higgs is favored by current data [1].

However, the MSSM is getting squeezed. The lightest Higgs is, at tree-level, lighter than the $Z$ boson. This mass range has been excluded by LEP-II, and would rule out the MSSM if not for the fact that large quantum corrections from the top sector can raise the Higgs mass to $130 \mathrm{GeV}$ - though only in the case of large $\tan \beta$ (the ratio of the VEV's of the two Higgses), $1 \mathrm{TeV}$ stop masses, and a maximal stop mixing angle [2]. The tension in the MSSM comes from the dual role played by the stops. On the one hand, the stops cut off the quadratic divergence of the top loops and thus should be no heavier than the electroweak scale to avoid fine-tuning. On the other hand, they must be heavy enough to generate a Higgs mass above the LEP-II bound.

The current experimental situation naturally leads one to consider extensions of the MSSM which relieve this tension. In particular, the tree-level bound on the Higgs mass is a direct consequence of the strength of the Higgs quartic coupling. In the MSSM, the Higgs quartic coupling comes only from the $D$-terms of the electroweak gauge groups and is therefore a fixed function of the (relatively small) electroweak gauge couplings.

The physical Higgs mass can be increased by enhancing the quartic coupling through extended gauge sector and/or new superpotential Higgs couplings [3]. This has been widely studied in the context of extra singlet or triplet Higgses [4]. For singlet and triplet soft masses of $1 \mathrm{TeV}$ the bound on the Higgs mass is found to be approximately $150 \mathrm{GeV}$ and $200 \mathrm{GeV}$ respectively. A thorough analysis of the electroweak precision and fine-tuning constraints in both types of models would be worthwhile.

Such models are limited in the bound on the Higgs mass by requiring perturbativity of all couplings up to the GUT scale. The issue: every contribution to the Higgs quartic coupling is infrared free. Couplings run from an already perturbative value at the GUT scale to smaller values near the weak scale. Thus we argue that in order to significantly increase the bound, the Higgs must be charged under an asymptotically-free group. This will require extending the gauge group of the MSSM.

The additional contribution to the quartic potential here is the $D$-term of the asymptotically free group. When the full gauge group breaks down to that of the standard model just above the weak scale, this new $D$-term would quickly decouple in the supersymmetric limit. Therefore, the field responsible for breaking the gauge symmetry must have a supersymmetry-breaking mass at or above the breaking scale. The effect is to retain the $D$ term in the potential at low energies. To avoid large unwanted contributions to electroweak precision measurements from the new gauge bosons, the breaking scale, and therefore the soft mass, should be in the multi-TeV range.

In our scenario, supersymmetry breaking can be much larger than the weak scale while retaining naturalness due to the (amazing) properties of $D$-terms: 
- After integrating out the field which breaks the gauge symmetry at the multi-TeV scale, we have an effective hard breaking of supersymmetry in the quartic sector of the model while the gauge and top sectors are still supersymmetric (broken softly). There is no one-loop quadratically divergent contribution to the Higgs mass parameter from the top sector cut off by this higher scale, but there is one proportional to the additional quartic term.

- While breaking a gauge symmetry in a non-supersymmetric way would in principle produce both quartic and mass terms for the Higgs fields from the $D$-term, a VEV in a $D$-flat direction leaves all fields without VEVs at that scale (e.g., the Higgs) massless at tree-level.

- The multi-TeV soft mass for the breaking field feeds into the Higgs mass renormalization group equations only at two loops and does not destabilize the weak scale. In addition, the breaking field quantum numbers typically disallow any renormalizable superpotential couplings to MSSM fields.

\section{Warm-up: $U(1)_{x}$}

Before we present our model we present a warm-up version with an extra $U(1)$ in which the non-decoupling of the $D$-term is in effect, though the group is of course non-asymptotically free. We then present a model with an asymptotically-free $S U(2)$. The dominant constraint on this model comes from electroweak precision measurements and the desire for naturalness in couplings.

Now take the MSSM and gauge a $U(1)_{x}$. A simple choice of charges is the $\tau^{3}$ generator of $S U(2)_{R}$, namely $Q, U^{c}, D^{c}, L, E^{c}$ have charges $0,-\frac{1}{2},+\frac{1}{2}, 0,+\frac{1}{2}$ respectively, $\bar{H}, H$ have charges $\pm \frac{1}{2}$ and three generations of right-handed neutrinos $N^{c}$ are added with charges $-\frac{1}{2}$ to cancel the $U(1)_{x}^{3}$ anomaly.

In addition to the MSSM $D$-terms,

$$
\frac{g^{2}}{8}\left(H^{\dagger} \sigma^{a} H-\bar{H} \sigma^{a} \bar{H}^{\dagger}+\ldots\right)^{2}+\frac{g_{Y}^{2}}{8}\left(|\bar{H}|^{2}-|H|^{2}+\ldots\right)^{2},
$$

we have an additional contribution to the Higgs potential coming from the new $U(1)_{x}$ $D$-term:

$$
\frac{g_{x}^{2}}{2}\left[\frac{1}{2}|\bar{H}|^{2}-\frac{1}{2}|H|^{2}+q|\phi|^{2}-q\left|\phi^{c}\right|^{2}+\ldots\right]^{2}
$$

where the ellipsis represents the rest of the charged MSSM scalars. The $\phi, \phi^{c}$ fields, responsible for breaking $U(1)_{x}$, are uncharged under the MSSM gauge group and have charges $\pm q$ under $U(1)_{x}$. Their scalar potential comes from the superpotential $\mathcal{W}=\lambda S\left(\Phi \Phi^{c}-w^{2}\right)$ and a soft mass $m_{\phi}^{2}$, giving a potential

$$
V_{\phi}=\lambda^{2}|\phi|^{2}\left|\phi^{c}\right|^{2}-B \phi \phi^{c}+\text { h.c. }+m_{\phi}^{2}\left(|\phi|^{2}+\left|\phi^{c}\right|^{2}\right),
$$

where $B \equiv \lambda w^{2}$ and all couplings are made real and positive from field redefinitions (except

$m_{\phi}^{2}$ which is automatically real and taken to be positive). We assume $\phi$ and $\phi^{c}$ soft masses are the same due to ultraviolet dynamics. 
For $B>m_{\phi}^{2}$, we have $\langle\phi\rangle^{2}=\left\langle\phi^{c}\right\rangle^{2}=\left(B-m_{\phi}^{2}\right) / \lambda^{2}$. Taking $B \gg v^{2}$, we integrate out the $\phi$ fields at tree-level and find an extra contribution to the MSSM Higgs potential:

$$
\frac{g_{x}^{2}}{2}\left(\frac{1}{2}|\bar{H}|^{2}-\frac{1}{2}|H|^{2}\right)^{2} \times\left(1+\frac{M_{Z^{\prime}}^{2}}{2 m_{\phi}^{2}}\right)^{-1},
$$

where $M_{Z^{\prime}}=2 q g_{x}\langle\phi\rangle$. In order to maximize the contribution to the Higgs mass, we would like a large but perturbative $g_{x}$ and a small $U(1)_{x}$ gauge boson mass compared to $m_{\phi}$. At the same time we don't want a soft mass so large that it destabilizes the weak scale.

Electroweak symmetry breaking occurs under the same conditions as in the MSSM. The adjusted tree-level bound for the CP-even Higgs mass is

$$
m_{h^{0}}^{2}<\left(\frac{g^{2}}{2}+\frac{g^{\prime 2}}{2}+\frac{g_{x}^{2}}{2}\left(1+\frac{M_{Z^{\prime}}^{2}}{2 m_{\phi}^{2}}\right)^{-1}\right) v^{2} \cos ^{2} 2 \beta,
$$

where the inequality is saturated in the "decoupling limit" when the CP-odd Higgs mass $m_{A}^{2}$ is much larger than $m_{h^{o}}^{2}$.

Electroweak precision measurements put a lower limit on the $Z^{\prime}$ mass as a function of its couplings. There are oblique corrections at order $v^{2} /\langle\phi\rangle^{2}$ to the $Z$ boson mass through its mixing with the $Z^{\prime}$, and non-oblique corrections to the $Z$ coupling to right-handed fermions at the same order. We perform a global fit to the low energy data (see [5] for details as to how the fit is implemented) provides the $95 \%$ C.L. $q\langle\phi\rangle \gtrsim 2 \mathrm{TeV}$, whereas the bound on the 4-lepton contact-interaction from LEP-II [1] is $q\langle\phi\rangle \gtrsim 3.75 \mathrm{TeV}$.

We therefore take the following example parameters:

- $\alpha_{x} \equiv g_{x}^{2} / 4 \pi=1 / 35$ at a few TeV. The beta-function coefficient for the gauge coupling $g_{x}$ is $b_{x}=7+2 q^{2}$. For the value $q=1 / 2$, the coupling runs semi-perturbatively at the $\operatorname{GUT}$ scale (i.e., $\left.\alpha_{x}\left(\Lambda_{G U T}\right) \sim 1\right)$.

- A $Z^{\prime}$ mass of $2.2 \mathrm{TeV}(\mathrm{q}=1 / 2)$, just above the current LEP lower bound.

- $m_{\phi}=6.6 \mathrm{TeV}$ at low energies. One loop corrections to the Higgs mass parameter from the supersymmetry breaking are finite and relatively small $(<250 \mathrm{GeV})$. The two-loop RGE contribution from $m_{\phi}^{2}$ is smaller.

The superpotential coupling $\lambda$ stays perturbative throughout the range of scales for this choice of parameters (without fine-tuning the value of $B$ ). The tree-level prediction for the Higgs mass can be computed from equation 2.5. In the decoupling limit with large $\tan \beta$, we find $m_{h^{0}}=116 \mathrm{GeV}$, consistent with the electroweak fits. The top-stop contribution to the one-loop effective potential results in an actual Higgs mass which is larger than this value. Thus for most of the parameter space consistent with other direct SUSY searches, the current direct search bound on the Higgs mass is satisfied.

Unification of the standard three gauge couplings is still predicted to the percent level as the new $U(1)_{x}$ coupling affects the running only at two loops.

We have presented a model which exemplifies a extra $D$-term contribution to the quartic potential of the Higgs. Since this section is intended as a warm-up, we have 
neither included the effects of kinetic mixing of this $U(1)$ with hypercharge, ${ }^{1}$ nor have we given details of how $D$-flatness is protected in the ultraviolet. Kinetic mixing will mix the $U(1)_{x}$ and $U(1)_{Y} D$-terms and can be cancelled by a suitable counterterm. If $\left(m_{\phi}^{2}-m_{\phi^{c}}^{2}\right) / \lambda \gtrsim 300 \mathrm{GeV}$ then the $U(1)_{x} D$-term generates a tree-level mass for the Higgs field which introduces $\gtrsim 10 \%$ fine-tuning into the Electroweak VEV. In the models below, neither of these points are relevant.

\section{An extra $S U(2)$}

In place of an extra $U(1)$, we now gauge an extra $S U(2)$ group. The standard-model fields are charged under $S U(2)_{1}$ as the normal weak group and there is an additional group $S U(2)_{2}$. To break the $S U(2)_{1} \times S U(2)_{2}$ to the diagonal subgroup we add an extra bidoublet $\Sigma$ which transforms as a $(2, \overline{2})$. Above the scale of diagonal symmetry breaking, the $S U(2)_{1} \times S U(2)_{2} D$-term is

$$
\frac{g_{1}^{2}}{8}\left(\operatorname{Tr}\left[\Sigma^{\dagger} \sigma^{a} \Sigma\right]+H^{\dagger} \sigma^{a} H-\bar{H} \sigma^{a} \bar{H}^{\dagger}+\ldots\right)^{2}+\frac{g_{2}^{2}}{8}\left(\operatorname{Tr}\left[\Sigma \sigma^{a} \Sigma^{\dagger}\right]\right)^{2} .
$$

The superpotential $\mathcal{W}=\lambda S\left(\frac{1}{2} \Sigma \Sigma+w^{2}\right)$ with an additional soft-mass $m^{2}$ for $\Sigma$ leads to the scalar potential

$$
V_{\Sigma}=\frac{1}{2} B \Sigma \Sigma+\text { h.c. }+m^{2}|\Sigma|^{2}+\frac{\lambda^{2}}{4}|\Sigma \Sigma|^{2} .
$$

Here, $\Sigma \Sigma$ is contracted with two epsilon tensors and $B=\lambda w^{2}$. For suffiently large $B, \Sigma$ acquires a $\mathrm{VEV},\langle\Sigma\rangle=u \mathbf{1}$, with $u^{2}=\left(B-m^{2}\right) / \lambda^{2}$, which breaks $S U(2)_{1} \times S U(2)_{2}$ to the diagonal subgroup. The minimum lies in a $D$-flat direction, leaving both Higgs fields massless.

Under the remaining $S U(2), \Sigma$ contains a complex triplet, $T$, along with a complex singlet. Integrating out the real part of the heavy triplet at tree-level gives the effective Higgs potential below the triplet mass,

$$
\begin{aligned}
& \frac{g^{2}}{8} \Delta\left(H^{\dagger} \vec{\sigma} H-\bar{H} \vec{\sigma} \bar{H}^{\dagger}\right)^{2}+\frac{g_{Y}^{2}}{8}\left(|\bar{H}|^{2}-|H|^{2}\right)^{2}, \\
& \text { with } \Delta=\frac{1+\frac{2 m^{2}}{u^{2}} \frac{1}{g_{2}^{2}}}{1+\frac{2 m^{2}}{u^{2}} \frac{1}{g_{1}^{2}+g_{2}^{2}}} \text { and } \frac{1}{g^{2}}=\frac{1}{g_{1}^{2}}+\frac{1}{g_{2}^{2}} .
\end{aligned}
$$

The MSSM $D$-term is recovered in the limit $u^{2} \gg m^{2}$ (no SUSY breaking), for which SUSY protects the $D$-term below the gauge-breaking scale.

As in the $U(1)$ case, electroweak symmetry breaking occurs under the same conditions as in the MSSM. We find the tree-level $W$ and $Z$ masses are corrected by the same relative amount, $\left(1-g^{4} v^{2} / 2 g_{2}^{4} u^{2}+\ldots\right)$ while the tree-level Higgs mass satisfies

$$
m_{h^{o}}^{2}<\frac{1}{2}\left(g^{2} \Delta+g_{Y}^{2}\right) v^{2} \cos ^{2} 2 \beta
$$

\footnotetext{
${ }^{1}$ We thank Hitoshi Murayama for reminding us of this point.
} 
To maximize the upper bound, $\Delta$ should be made as large as possible by sending $g_{1} \rightarrow \infty$, $g_{2} \rightarrow g$ and $m^{2} \gg u^{2}$ by as much as possible without introducing fine-tuning.

Precision electroweak constraints were analyzed in [7] resulting in the $95 \%$ C.L. constraint $(1 / 2)\left(g / g_{2}\right)^{4}(v / u)^{2} \leq 2.1 \times 10^{-3}$. However, our setup has an additional contribution to the oblique parameter $T$ due to a small triplet VEV. This results in a contribution $\Delta T \sim\left(4 \pi / s_{W}^{2} c_{W}^{2}\right)\left(g_{1}^{4} / g^{4}\right)\left(M_{W}^{2} u^{2} / M_{T}^{4}\right)$, where $M_{T}$ is the triplet mass. The triplet VEV and larger $m_{h^{0}}$ partially compensate each other in the $\Delta T$ piece of the electroweak fit.

A sample point in which perturbative unification is achieved with the right matter content at the GUT scale (see below for more details) is $g_{1}(u)=1.05$ and $g_{2}(u)=0.83$. Precision electroweak constraints and fine-tuning bounds are avoided for $m=2 u=3.3$ $\mathrm{TeV}$, which implies $m_{Z^{\prime}}=m_{W^{\prime}}=2.5 \mathrm{TeV}$. For this sample point, $\Delta=2.3$ and $m_{h^{0}}=129$ $\mathrm{GeV}$ at tree-level in the large $\tan \beta$ and decoupling limits. Again this Higgs mass, while large enough to comfortably evade the LEP-II bounds, is consistent with the electroweak fits.

The size of $g_{1}$ (and therefore $\Delta$ ) in the $S U(2)$ scenario was limited by its large positive beta-function coefficient. One can ameliorate this situation by instead dividing the matter between the two $S U(2)$ groups such that $g_{1}$ runs asymptotically-free and is thus larger at the weak scale - leading to a larger value of $\Delta$. We consider a non-universal model with the Higgses and third family charged under $S U(2)_{1}$, while the first two families are charged under $S U(2)_{2}$.

Yukawa couplings for the first two generations can be generated by adding a massive Higgs-like pair of doublets $\bar{H}^{\prime}, H^{\prime}$, that are charged under $S U(2)_{2}$. They couple to the first two generations via Yukawa-type couplings and mix with the regular Higgses via superpotential operators such as $\lambda^{\prime} \bar{H} \Sigma H^{\prime}$. A supersymmetric mass $\left.\mu_{H^{\prime}}\right\rangle\langle\Sigma\rangle$ for the new doublets generates naturally small Yukawa couplings for the first two generations at low energies.

The constraints on a non-universal model, however, are more severe, as there are treelevel non-oblique corrections to the third family couplings [8]. We fit the precision data, including the additional contribution to $\Delta T$ from the triplet VEV and find the (95\% C.L.) constraint on $u$ as a function of $g / g_{2}$. The strongest constraints occur for $g^{2} / g_{2}^{2} \rightarrow 0,1$.

We take the following example parameters:

- $g_{1}(u)=1.80, g_{2}(u)=.70$, inspired by a GUT with $g_{1}\left(\Lambda_{G U T}\right)=.97$. Additional spectator fields (see the full description at the end of the section for details) are included in the running to aid in unification.

- $u=2.4 \mathrm{TeV}$, above the lower limit from electroweak constraints, giving $M_{W^{\prime}}, M_{Z^{\prime}} \sim$ $4.5 \mathrm{TeV}$.

- $m=10 \mathrm{TeV}$. One-loop finite corrections to the Higgs mass parameter from supersymmetry breaking are $<300 \mathrm{GeV}$ whereas two-loop RGE contributions can be somewhat larger if one assumes high-scale supersymmetry breaking. 
We find $\Delta=6.97$ and $m_{h}=214 \mathrm{GeV}$ at tree-level in the large $\tan \beta$ and decoupling limits. Loop corrections to the effective potential from the top sector and the additional physics will make a relatively small shift in the tree-level result.

Since $S U(2)_{1}$ is asymptotically free, we can push $\alpha_{1}(u)$ to the perturbative limit, $\alpha_{1}(u)=1$, by adjusting its high-scale value. Electroweak precision constraints for this $g / g_{2}$ require $u \gtrsim 3.1 \mathrm{TeV}$, while fine-tuning at the GUT-scale increases as we tune the confinement scale and $u$ to coincide. For $g_{1}(u)=3.75$ and $g_{2}(u)=.66$, we choose $g_{1}\left(\Lambda_{G U T}\right)=1.1$, tuned to be within $1 \%$ of its critical value. For $m=10 \mathrm{TeV}$, we find $\Delta \sim 20$ and $m_{h} \sim 350$ $\mathrm{GeV}$ in the large $\tan \beta$ and decoupling limits. While the large Higgs mass gives a small positive contribution to the $\mathrm{S}$-parameter and a large negative contribution to the T-parameter, this effect is offset in the global fit by a positive T-parameter contribution from the small triplet VEV. Quantum contributions to the Higgs mass parameter are of order $1.3 \mathrm{TeV}$ which represents a fine-tuning of around $7 \%$.

One interesting feature of this model is that because there is a gauge coupling larger than that of $S U(3)$ color, the top yukawa "fixed point" has a much larger value than in the MSSM. In this sense, a favorable region of parameter space includes some of $\tan \beta<1$ which can both be consistent with the Higgs mass bound and avoid a Landau pole for the top Yukawa.

This model can also be made consistent with gauge coupling unification. The full group $S U(3)_{c} \times S U(2)_{1} \times S U(2)_{2} \times U(1)_{Y}$ can be embedded in $S U(5) \times S U(5)$ [9] broken by a bi-fundamental field at the GUT scale with a vev $\langle\Xi\rangle=\operatorname{diag}\{M, M, M, 0,0\}$. Gauge coupling unification is predicted (with theoretical uncertainty beyond one-loop) because the standard model gauge couplings are only a function of the diagonal gauge coupling. At one loop, one can track the diagonal $S U(2)$ through its beta-function coefficient $b$ as it is the sum of those of the two $S U(2)_{i}$. It receives an extra -6 from the additional triplet of gauge bosons. We include two triplets charged under $S U(2)_{2}$ which, with the diagonalbreaking $\Sigma$ field, contribute +6 to the diagonal beta function. We have also added an additional vector-like pair of triplets to effectively complete a 5 and $\overline{5}$ with the extra pair of Higgs-like fields (however, they should be from a split multiplet as they must not share the Yukawa couplings with the doublets due to proton decay). With these additions, the $S U(2)$ model achieves the same unification accuracy as in the MSSM at one loop. Though there is a gauge coupling that gets relatively strong, its two-loop effect is still small as $g_{1}$ is quite perturbative for nearly all of the running.

\section{Conclusions and Outlook}

The point of this paper is to show that asymptotically-free gauge extensions of the MSSM can produce significant contributions to the Higgs quartic coupling - and therefore the physical Higgs mass - without destabilizing the weak scale. Breaking extra gauge groups in the multi-TeV range with a soft mass for the breaking field at the same scale leaves a nondecoupling contribution to the Higgs quartic potential. Because of the $D$-term structure, there are no log-enhanced one-loop contributions to the Higgs soft mass and thus even after running from high-scales, the electroweak scale remains natural. 
While technically natural, what could be the source of this higher scale (few - 10 $\mathrm{TeV}$ ) which is necessary for the extra gauge breaking and the breaking-field's soft mass? In fact, models of anomaly-mediated supersymmetry breaking [10] and gaugino-mediated supersymmetry breaking [11] provide such a scale. Specifically, $\mu$-like terms are enhanced by a loop factor in the former, while soft masses for bulk scalars are enhanced by a volume factor in the latter.

In addition, there currently exists in the literature supersymmetric models which make use of extended gauge sectors. For example, extra gauge groups are used to avoid negative squared masses for sleptons in models of anomaly-mediated supersymmetry breaking [12, 13]. It would be interesting to calculate how much the quartic coupling can be enhanced in these models. If Higgs mass bounds increase, they may turn out to be more natural than the MSSM.

An interesting question to ask is what happens if we allow the $S U(2)_{1}$ coupling to blow up at the preferred breaking scale. From arguments involving "complementarity" 14, 15, we speculate that the composite theory in the infrared mimics the weakly coupled theory in the Higgs phase. The Higgs and the third generation would be composite and strongly coupled and therefore arbitrary Higgs masses (consistent with unitarity bounds) would appear possible. One remarkable property of this model would be that gauge coupling unification would still only be affected at the few percent level, as in 16. Of course it is crucial that supersymmetry breaks at the same scale so a severe fine-tuning of scales would be required. It would be interesting to see to what extent that accident could have a dynamical origin.

The natural regions of parameter space in these models leave behind extra gauge bosons with masses of order 2-5 TeV. Due to the stronger gauge coupling, these may be accessible at the LHC. Thus, if superpartners are discovered, a search for an extended gauge sector could be fruitful even if the Higgs mass is below $130 \mathrm{GeV}$.

Note: Half a plenary talk at SUSY02 by L. Randall (who cited work in progress by N . Arkani-Hamed, N. Weiner and herself) was devoted to an idea involving non-decoupling $D$-terms [17].

\section{Acknowledgments}

The authors have benefitted from discussions and general abuse from J.R. Espinosa A. Nelson, M. Quiros, and C.E.M. Wagner, and are grateful to the Aspen Center for Physics, at which much of this work was envisioned. A.D., D.E.K. and P.B. are supported by NSF Grants P420D3620414350 and P420D3620434350. Fermilab is operated by Universities Research Association Inc. under contract no. DE-AC02-76CH02000 with the DOE.

\section{References}

[1] D. Abbaneo et al. [ALEPH Collaboration], arXiv:hep-ex/0112021.

[2] Y. Okada, M. Yamaguchi and T. Yanagida, Prog. Theor. Phys. 85, 1 (1991); M. Carena, M. Quiros, and C. E. M. Wagner, Nucl. Phys. B 461, 407 (1996) [arXiv:hep-ph/9508343]; 
H. E. Haber, R. Hempfling, and A. H. Hoang, Z. Phys. C 75, 539 (1997)

[arXiv:hep-ph/9609331]; J. R. Espinosa and I. Navarro, Nucl. Phys. B 615, 82 (2001)

[arXiv:hep-ph/0104047]. K. Tobe and J. D. Wells, Phys. Rev. D 66, 013010 (2002)

[arXiv:hep-ph/0204196].

[3] H. E. Haber and M. Sher, Phys. Rev. D 35, 2206 (1987). M. Drees, Phys. Rev. D 35, 2910 (1987). K. S. Babu, X. G. He and E. Ma, Phys. Rev. D 36, 878 (1987). J. R. Espinosa and M. Quiros, Phys. Lett. B 279, 92 (1992). J. R. Espinosa and M. Quiros, Phys. Lett. B 302, 51 (1993) [arXiv:hep-ph/9212305]. M. Cvetic, D. A. Demir, J. R. Espinosa, L. L. Everett and P. Langacker, Phys. Rev. D 56, 2861 (1997) [Erratum-ibid. D 58, 119905 (1998)] [arXiv:hep-ph/9703317];

[4] J. R. Espinosa and M. Quiros, Phys. Rev. Lett. 81, 516 (1998) [arXiv:hep-ph/9804235].

[5] D. Choudhury, T. M. Tait and C. E. Wagner, Phys. Rev. D 65, 053002 (2002) [arXiv:hep-ph/0109097].

[6] S. P. Martin and M. T. Vaughn, Phys. Rev. D 50, 2282 (1994) [arXiv:hep-ph/9311340].

[7] R. S. Chivukula, H. J. He, J. Howard and E. H. Simmons, arXiv:hep-ph/0307209.

[8] R. S. Chivukula, E. H. Simmons and J. Terning, Phys. Rev. D 53, 5258 (1996) [arXiv:hep-ph/9506427]; D. J. Muller and S. Nandi, Phys. Lett. B 383, 345 (1996) [arXiv:hep-ph/9602390]; E. Malkawi, T. Tait and C. P. Yuan, Phys. Lett. B 385, 304 (1996) [arXiv:hep-ph/9603349]; E. Malkawi and C. P. Yuan, Phys. Rev. D 61, 015007 (2000) [arXiv:hep-ph/9906215].

[9] G. D. Kribs, 10th International Conference on Supersymmetry and Unification of Fundamental Interactions (SUSY02), Hamburg, Germany, 17-23 Jun 2002

[10] L. Randall and R. Sundrum, Nucl. Phys. B 557, 79 (1999) [arXiv:hep-th/9810155]. G. F. Giudice, M. A. Luty, H. Murayama and R. Rattazzi, JHEP 9812, 027 (1998) [arXiv:hep-ph/9810442].

[11] D. E. Kaplan, G. D. Kribs and M. Schmaltz, Phys. Rev. D 62, 035010 (2000) [arXiv:hep-ph/9911293]. Z. Chacko, M. A. Luty, A. E. Nelson and E. Ponton, JHEP 0001, 003 (2000) [arXiv:hep-ph/9911323].

[12] N. Arkani-Hamed, D. E. Kaplan, H. Murayama and Y. Nomura, JHEP 0102, 041 (2001) [arXiv:hep-ph/0012103]; B. Murakami and J. D. Wells, Phys. Rev. D 68, 035006 (2003) [arXiv:hep-ph/0302209].

[13] A. E. Nelson and N. T. Weiner, arXiv:hep-ph/0210288.

[14] E. H. Fradkin and S. H. Shenker, Phys. Rev. D 19, 3682 (1979).

[15] S. Raby, S. Dimopoulos and L. Susskind, Nucl. Phys. B 169, 373 (1980).

[16] S. Dimopoulos and D. E. Kaplan, arXiv:hep-ph/0203001.

[17] L. Randall, talk at The 10th International Conference on Supersymmetry and Unification of Fundamental Interactions (SUSY02)", Hamburg, Germany, 17-23 Jun 2002, http://www.desy.de/susy02/ . 